\title{
Reconstruction of Mandibular Defect by Free Re-Vascularized Fibula Graft: A Case Report
}

\author{
Quazi Billur Rahman', Mahmudur Rahman', Showkat Mamun', Munjur Iqbal', Binay Kumar Das ${ }^{3}$ \\ ${ }^{1}$ Associate Professor, Department of Oral \& Maxillofacial Surgery, Faculty of Dentistry, BSMMU, ${ }^{2}$ MPH (Student), NIPSOM, ${ }^{3}$ Medical Officer, \\ Department of Oral \& Maxillofacial Surgery, Dhaka Dental College
}

\begin{abstract}
:
Background: In maxillofacial surgery tumor ablation often causes continuity defect of mandible which results anatomical and functional morbidity of the patient. The reconstruction of the mandibular defect is mandatory to restore the oral function and speech. Various methods of immediate reconstruction are implemented by different authors time to time including autogenous non vascularized bone graft, allogenic bone graft, auto frozen mandible or reconstruction plates and others. Each has its own advantages and disadvantages including donor site morbidity, failure and others. The purpose of the present case report is to establish micorvascular free fiblula is as a better option to other methods in immediate reconstruction of mandibular continuity defect. Objective: Anatomical, functional and esthetic rehabilitation of patients after mandibular resection Method: Revascularization of free fibula graft by microvascular anastomosis of paroneal artery with facial artery at the segmental defect site of mandible. Result: Remarkable contour, cosmesis and early functional rehabilatation of the patient. Conclusion: Microvascular reconstruction with fibula is the better option for defect correction and early rehabilitation in patients with mandibular continuity defect.
\end{abstract}

Key word: Mandible, defect, reconstruction, microvascular technique, fibula graft

[BSMMU J 2008; 1(1): 35-38]

\section{Introduction:}

Tumor, malignancy, radionecrosis, cystic lesion, trauma, infection or congenital anomalies may be reasons for mandibular defects. Among them the benign lesion affect most commonly. ${ }^{1-3}$ The management of mandibular continuity defect has changed in the last decade. The most frequently used technique for reconstruction of extended defect is the transfer of vascularized osseous free graft. The fibula, scapula, rib and the illiac crest are the preferred donor-sites for reconstruction. ${ }^{4,5}$ It is essential to establish bone viability after revascularization of the graft . Lack of vitality as a result of vascular occlusion either arterial or venous can result in graft necrosis, bone resorption and poor healing.
Incidence of ameloblastoma in mandible is one of the most common causes of mandibular defect. Goal of the surgery, include resection of mandible and immediate reconstruction to maintain the function- speech, mastication \& deglutation, facial contour and oral competence. ${ }^{6}$ However, the best option to reconstruct mandibular continuity defect has not yet been satisfactorily resolved and represents a challenge for oral and maxillofacial surgeons.

\section{Case History:}

A 18 years old Bangladeshi woman came to the OPD of oral \& maxillofacial surgery Dept. of Bangabandhu Sheikh Mujib Medical University with 2 years history of slowly growing lesion at the left side of the lower face.
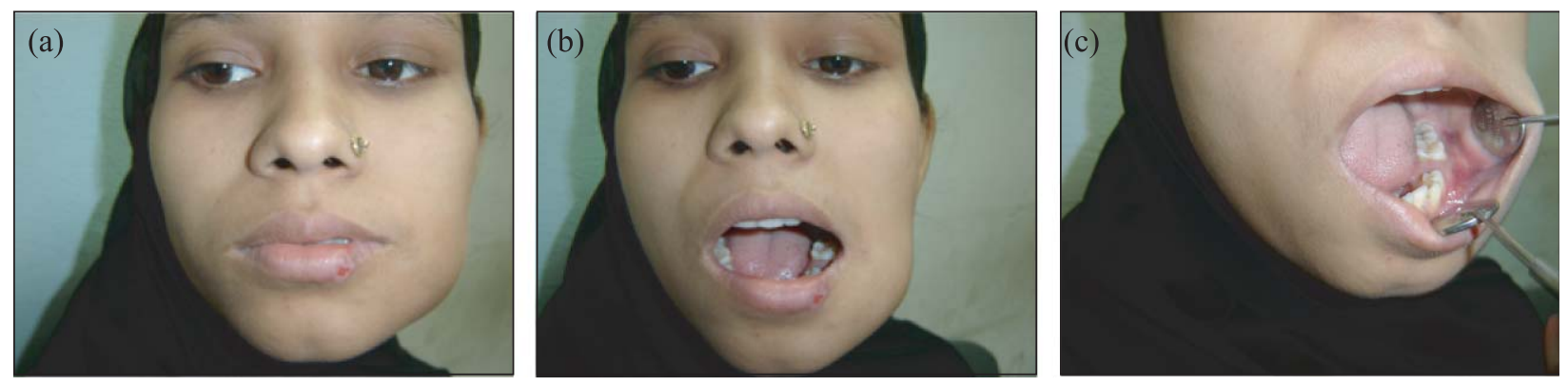

Fig.1: Preoperative view of the patient with close (a) \& open (b) (c) mouth.

Address of correspondence to: Dr. Quazi Billur Rahman, Department of Oral \& Maxillofacial Surgery, Faculty of Dentistry, Bangabandhu Sheikh Mujib Medical University (BSMMU), Dhaka 
The lesion was non-tender and there was no regional lymphadenopathy with no motor or sensory functional deficit. She was otherwise fit and was not medically compromised. On examination the size of the lesion was

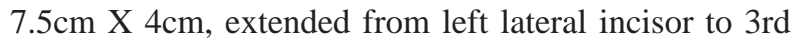
molar of mandible. Cortical expansion was marked on the buccal side but lingual expansion of the cortex was minimal. There was no intra oral or extra oral persistent sinus or discharge. The remainder of the oral cavity was unremarkable.

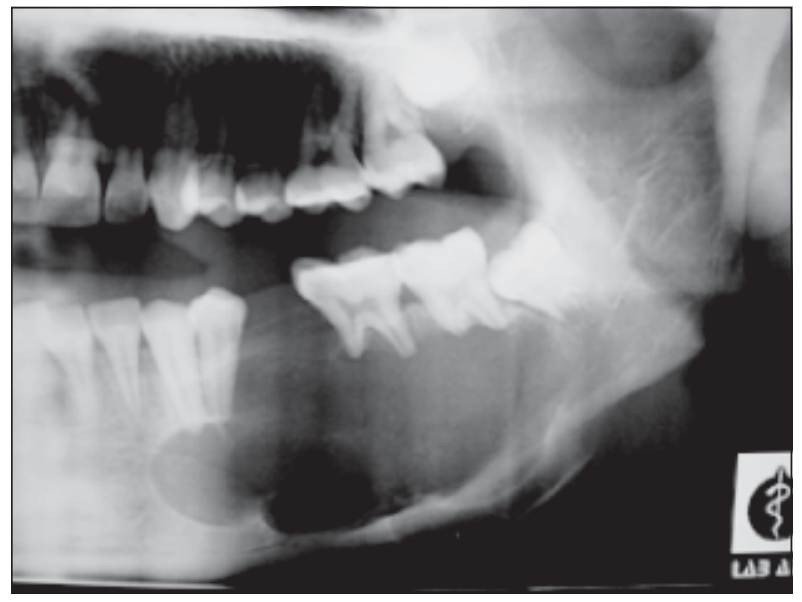

Fig.-2: Preoperative Orthopantamogram

Orthopantamograph revealed a multilocular radiolucent lesion involving the body of the madible from left lateral incisor to third molar. Clinically, radiologically and histologically it was diagnosed as a case of ameloblastoma.

\section{Methods:}

She underwent surgery as a case of ameloblastoma with the treatment plan of a wide excision of the lesion followed by immediate reconstruction with revascularized fibula graft for the first time in our country.
Two teams were involved in the surgery. One team extirpated the tumor and the other team simultenously harvested the fibula. A left submandibular approach was used to expose the lesion. Subperiosteal dissection was carried out to expose the tumor and it was resected with 1 cm healthy bone on each side. The facial artery and the vein were secured with vascular clips for future vascular anastomosis. On the donor site the other team simultaneously exposed the fibula utilizing lateral approach.

A line was drawn from the fibular head to the lateral malleolus indicating the submascular and subcutenous course of fibula. Two markings were made on the line. First was $7 \mathrm{~cm}$ distal from the fibular head which indicate approximate insertion of peroneal vessels within the intermascular septum. Second marking was $14 \mathrm{~cm}$ distal from the fibular head indicating the approximate location of nutrient vessels. A curvilinear incision is then make along the lateral border of the peroneal muscles. The posterior intermuscular septum was identified separating the peroneal muscle from soleus muscle. The septum is separated from its attachments to the fibula along its posterior border. Dissection next proceeds anteriorly toward the anterior intermuscular septum which seperates the peroneal muscle from the extensor muscle. Extraperiosteal dissection proceeds and about $1 \mathrm{~cm}$ cuff of flexor hallucis muscle with associated peroneal vessels was left attached to the fibula. The paroneal vessels were identified at the distal osteotomy site and were ligated. The paroneal vessel was identified to their origin from posterior tibial artery. The first osteotomy cut was done $4 \mathrm{~cm}$ distal to the nutrient vessel by gigli saw with $1 \mathrm{~cm}$ excess periosteum. The second osteotomy cut was done $4 \mathrm{~cm}$ proximal to the nutrient vessels with $1 \mathrm{~cm}$ excess periosteum also. The osseous tissue attached only by its
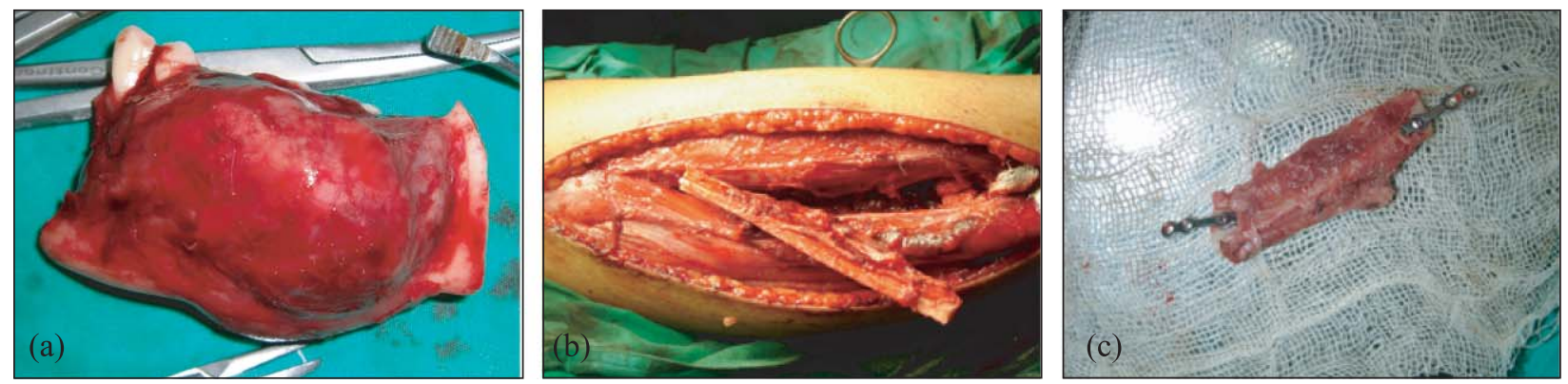

Fig.3: a) Excised tumour b) Harvesting of Fibula with patent paroneal vessels c) Adapted fibula graft with plates 


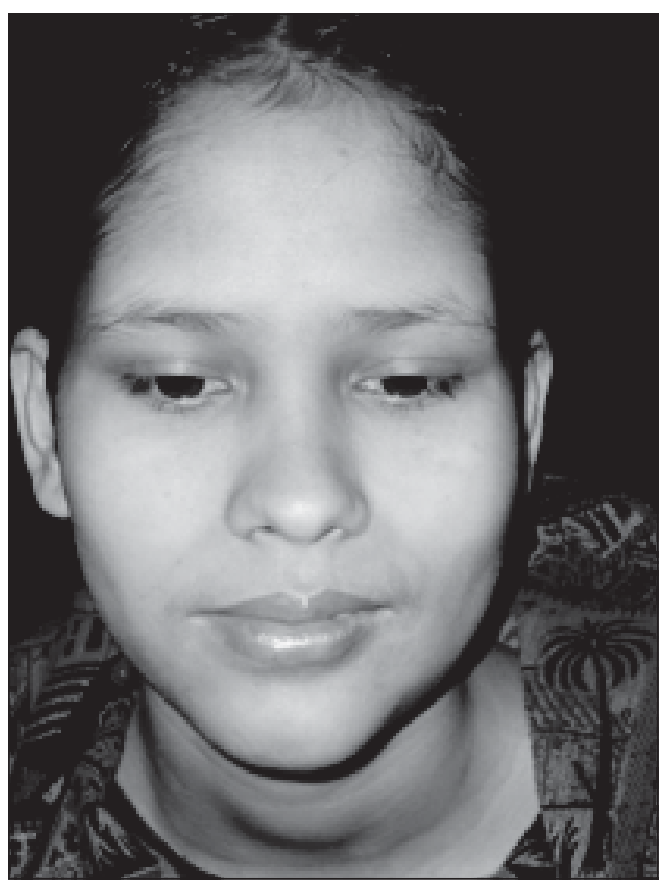

Fig.-4: Postoperative front view after 3 months. with good wound healing. She had an excellent recovery both functionally \& aesthetically. She was under in regular follow up in the department of oral and maxillofacial surgery. She was released after 10 days of surgery and cameto the first follow-up after one month of surgery. In first follow-up she was apparently better with no donor and recipient site complication both clinically and radiologically. In second follow-up three months (Fig.4) after the surgery the oral opening, closing and mastication was adequate. In radiological observation the graft was in proper position and significant amount of callous was formed with no complication with the donor site. The final follow-up (Fig.5) was given after one year which reveled excellent functional and cosmetic result. Radiologically the bony union was completed and no resorbtion of the graft was observed except two miniplates on either side but the medullary cavity of the graft was the isolating criteria from the graft and mandible..
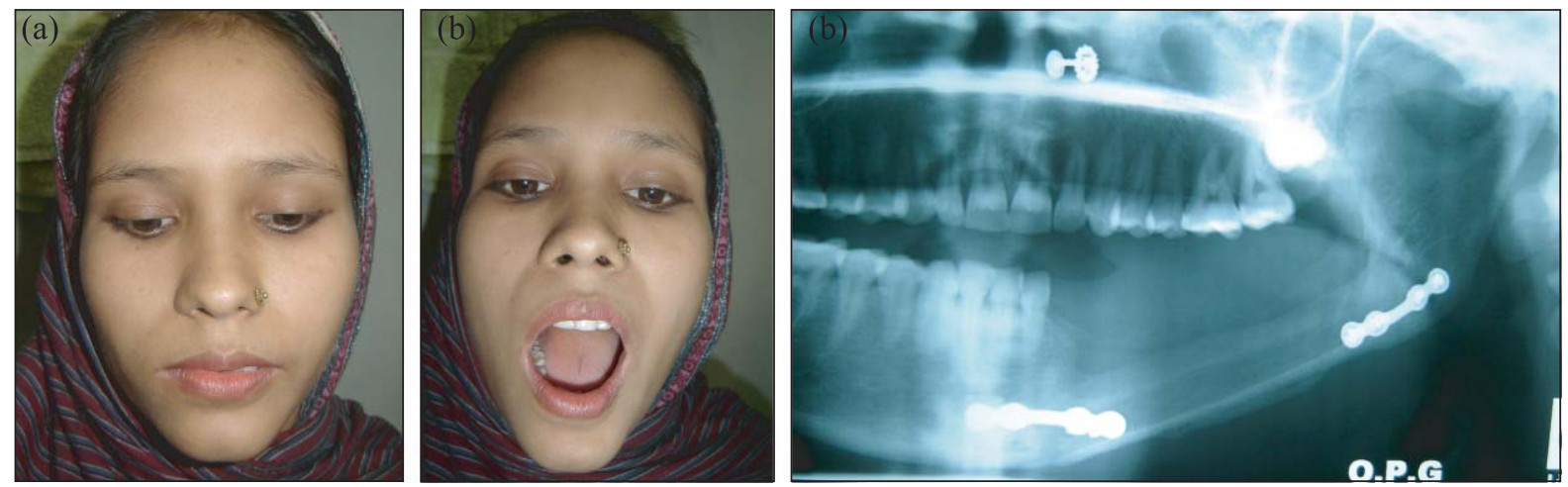

Fig.5: Follow up after One year a) Closed mouth b) Open mouth c) OPG after one year.

vascular pedicle was observed for balanced perfusion after deflating the torniquet. Then the proximal peroneal vessel was ligated and cut. The graft was then trimmed and adapted to the defect as per size and shape and stabilized by miniplate with screws. The end to end anastomosis was done in between paroneal and facial artery with 9/0 prolene under aided vision of loop in a convetional way. The clamps were released and patency of the anastomosed vessel was observed for a little period. Then the wound was closed in layers with water tight seal in the oral cavity.

\section{Follow up:}

She received broad spectrum antibiotic, analgesics for 10 days and antiseptic mouth wash for 14 days following surgery. The entire post operative period was uneventful

\section{Discussion}

Mandible plays an important role in airway protection, support for the tongue, muscles of the floor of the mouth, lower jaw dentition, articulation, deglutition, speech, respiration and facial aesthesis. Vascularised osseous free graft are used to good advantage in maxillofacial surgery for the reconstruction of mandibular defect following mandibular resection. The goal of the reconstruction areestablishment of mandibular continuity with acceptable cosmetic result, establishment of osseous alveolar base for further dental rehabilitation, correction of soft tissue defect. ${ }^{7}$ The surgeon has to balance his procedure to achieve best cosmetic appearance with reliable function. In order to achieve it one must restore bony continuity, facial contour, tongue mobility and speech. For restoration 
of the mandibular defect the use of autogenous bone is the preferred option. The other options are solely alloplastic materials or alloplast \& bone graft together. Alloplastic materials are in the form of stainless steel plates (alloy of iron, chromium, nickel) or vittalium (alloy of chromium, cobalt, molybdenum) ${ }^{8}$. Facial deformity, poor aesthetics, orocutaneous fistula was noted after mandibular reconstruction by other convetional methods due to lack of vascularity. ${ }^{10}$ But in our case these were not observed. The fibula provides the longest segment of bone with 20$30 \mathrm{~cm}$ available for harvest. In addition the segmental blood supply of the bone permits multiple osteotomy. The bone is also adequate width $\&$ height to allow placement of osseointigrated dental implants. Donor site morbidity with this graft is minimal unless the distal osteotomy site is within $6 \mathrm{~cm}$ of the ankle. In addition the location of the graft will allow simultenous harvest by a second team at the time of tumor resection. ${ }^{5,9}$ In our case $9 \mathrm{~cm}$ of fibular graft was used to reconstruct the mandible. There is a risk of peroneal nerve injury with resultant foot drop or weakness in planter flexion of the great toe which can be avoided with meticulous dissection. Advances in anastomotic technique, monitoring devices will add to its success.In the follow up period, orthopantamogram should be done routinely to assess bone resorption and every time compared with the immediate postoperative radiograph.

\section{References:}

1. Keszler A, Guliemotti MB, Dominguez F. Oral pathology in children: frequency, distribution, and clinical significance. Acta Odontal Latinoam. 1990; 5: 39-48.

2. Cordeiro PG. Disa JJ. Hidalgo DA. Hu OY. Reconstruction of the mandible with osseous free flaps a 10-year experience with 10 consecutive patients. Plast Reconstr Surg 1999; 104: 1314-20.

3. Foster RD. Anthony JP. Sharma A, Pogrel MA. Vascularized bone flaps versus nonvascularized bone grafts for mandibular reconstruction an outcome analysis of primary bony union and endosseous implant success. Head Neck 1999; 21: 66-71.

4. Urken ML. Weinberg H, Vickery C. Oromandibular reconstruction using microvascular composite free flaps. Arch. Otolaryngol Head Neck Surg. 1991; 117: 733-44.

5. Genden E, Haughey BH: Mandibular reconstruction by vascularized free tissue transfer. Am J Otolaryngol. 1996; 17: 21927.

6. Ritvik P. Mehta and Daniel G. Deschler. Mandibular reconstruction in 2004: an analysis of different techniques. Current Opinion in Otolaryngology \& Head and Neck Surgery 2004; 12: 288-293.

7. Urken ML. Buchbinder D. Chapter 86. In: Cummings CC editor. Oromandibular Reconstruction in otolaryngology-Head and Neck Surgery. St Louis: Mosby Year Book. 1998 p.1654-68.

8. Koch WM, Yoo GH. Goodstein ML. Advantages of mandibular reconstruction with the Titanium Hollow Screw Osseountegrating. Reconstruction Plate (THORP). Laryngoscope 1994; 104: 545-52.

9. Horiuchi K, Hattori A, Inada I. Mandibular reconstruction using the double barrel fibular graft. Microsurgery 1995; 16: 450-54.

10. Hidalgo DA, Pusic AL. Free-flap mandibular reconstruction: a 10-year follow-up study. Plast Reconstr Surg 2002; 110: 438-39 